\title{
Treinamento físico de paciente com a síndrome de Hallervorden- Spatz: um relato de caso
}

\section{Physical training of a patient with Hallervorden-Spatz syndrome: a case report}

\author{
Lícia Maria Belchior Almeidaํ. Luciana Dias Belchior \\ 1 Universidade Federal do Ceará (UFC), Fortaleza, Ceará, Brasil.
}

\section{RESUMO}

Introdução: A Síndrome de Hallervorden-Spatz é uma rara doença neurodegenerativa, autossômica recessiva, com alterações características do piramidalismo e extrapiramidalismo, além de distúrbios emocionais, sendo o diagnóstico caracterizado pela presença do sinal do olho do tigre, encontrado na ressonância magnética. Objetivo: Descrever o efeito da intervenção com treinamento físico na Síndrome de Hallervorden-Spatz por meio de um estudo de caso. O protocolo de exercícios físicos teve como objetivo reeducação da marcha, fortalecimento muscular de (membros superiores) MMSS e (membros inferiores) MMII, melhora no equilíbrio e expansão pulmonar. Metodologia: Tratou-se de um estudo de caso desenvolvido no Núcleo de Atenção Médica Integrada, de outubro a novembro de 2017, durante 60 minutos, total de 10 atendimentos. Resultados e discussão: Paciente J.R.G.S., 28 anos, sexo masculino, diagnosticado com síndrome de Hallervorden-Spatz (SHS) há 8 anos, e, há 3 anos, deu início ao treinamento físico, com alongamento, crioestimulação, estimulação elétrica funcional, plataforma vibratória, disco de propriocepção e barras paralelas, mostrando boa resposta ao tratamento, resultando na melhora da qualidade de vida. Conclusão: A intervenção proporcionou um resultado satisfatório ao paciente, o qual apresentou melhora no equilíbrio, na marcha, na coordenação e no ganho na amplitude de movimento.

Palavras-chave: Doenças raras. Prevenção terciária. Terapêutica. Equilíbrio postural.

\begin{abstract}
Introduction: Hallervorden-Spatz Syndrome is a rare neurodegenerative, autosomal recessive disease, with characteristic alterations of pyramidalism and extrapyramidalism, in addition to emotional disturbances, the diagnosis being characterized by the presence of the sign of the eye of the tiger, found on magnetic resonance imaging. Objective: To describe the effect of intervention with physical training on Hallervorden-Spatz Syndrome through a case study. The physical exercise protocol aimed at re-education of gait, muscle strengthening of upper limbs and lower limbs, improvement in lung balance and expansion. Methodology: This was a case study developed at the Center for Integrated Medical Care, from October to November 2017, for 60 minutes, total of 10 visits. Results and discussion: Patient JRGS, 28 years old, male, diagnosed with Hallervorden-Spatz syndrome (SHS) 8 years ago, and 3 years ago, started physical training, with stretching, cryostimulation, functional electrical stimulation, vibrating platform, proprioception disc and parallel bars, showing good response to treatment, resulting in improved quality of life. Conclusion: The intervention provided a satisfactory result to the patient, which showed improvement in balance, gait, coordination and gain in range of motion.
\end{abstract}

Keywords: Rare diseases. Tertiary prevention. Therapeutics. Postural balance.

Autor correspondente: Lícia Maria Belchior Almeida, Rua Coronel Nunes de Melo, 1127, Porangabuçu, Fortaleza, Ceará. CEP: 60430-275. Telefone: +5585 99696-0962. E-mail: liciamba@hotmail.com

Conflito de interesses: Não há qualquer conflito de interesses por parte de qualquer um dos autores.

Recebido em: 25 Mar 2020; Revisado em: 02 Out 2020; Aceito em: 27 Abr 2021. 


\section{INTRODUÇÃO}

A síndrome de Hallervorden-Spatz (SHS) é uma rara doença neurodegenerativa que se caracteriza por disfunções extrapiramidais e demência. Julius Hallervorden e Hugo Spatz foram os primeiros a descrevê-la, em 1922, como uma nova forma de degeneração familiar com características histológicas, nomeadas pela deposição de ferro. ${ }^{1}$

Herdada por herança genética autossômica recessiva, com $15 \%$ dos pacientes esporádicos. Ocorre em todas as raças, com frequência similar em ambos os gêneros. O início típico ocorre na infância, e, com o curso progressivo da doença, a maioria dos acometidos têm resultado fatal em 2 a 10 anos. A incidência é de 1-3/1.000.000. ${ }^{2}$

O acúmulo de ferro decorre da mutação da proteína pantotenato quinase (PKAN), mais comum no tipo 2 (PANK2), ${ }^{3}$ no cromossomo 20p13-p12.3. ${ }^{4}$

Não se sabe ao certo a etiologia dessa afecção. Sugere-se a peroxidação anormal e deficiência da cisteína dioxigenase, resultando no acúmulo de ferro no cérebro, especificamente nos glóbulos pálidos e substância negra mesencefálica. No entanto, o papel exato desse metal na etiologia da doença permanece desconhecida. A confirmação pode ser dada pelo estudo histopatológico ou pela ressonância magnética (RM), que apresenta o sinal do olho do tigre. ${ }^{5}$

As alterações são progressivas nos sistemas piramidais (espasticidade) e extrapiramidais (distonia, disartria, rigidez, e movimentos coreoatetósicos), com achados psiquiátricos associados (distúrbio cognitivo, da fala e agressividade). ${ }^{5}$

O tratamento farmacológico que responde melhor para o tremor são os agentes dopaminérgicos. Já anticolinérgicos ajudam na rigidez e tremor e são usados para os movimentos coreoatetósicos. Sintomas como sialorréia e disartria são problemáticos, utilizando-se brometo de metilscopolamina. Agentes utilizados para aliviar rigidez e espasticidade podem ser eficazes contra a disartria. A gastrostomia está indicada quando a nutrição por via oral não é possível. Injeções de toxina botulínica podem melhorar a distonia muscular. ${ }^{6}$

Um estudo propôs protocolo individualizado de tratamento baseado em treinamento de equilíbrio, de coordenação, de correção postural, de relaxamento da musculatura hipertônica e de expansibilidade pulmonar. $^{7}$

\section{MATERIAL E MÉTODOS}

Tratou-se de um estudo de caso de um paciente com diagnóstico clínico de síndrome de Hallervorden-Spatz (SHS), no Núcleo de Atendimento Médico Integrado (NAMI), em Fortaleza, durante dois meses, duas vezes por semana, durante uma hora, totalizando 10 atendimentos. O presente relato de caso foi aprovado no Comitê de Ética da Universidade de Fortaleza, sob o parecer de número 2.300.177.

\section{RESULTADOS E DISCUSSÃO}

Paciente J.R.G.S., 28 anos, gênero masculino, diagnosticado com síndrome de Hallervorden-Spatz (SHS). Apresenta consanguinidade positiva, porém, sem relatos da doença na família. Não foram evidenciados surtos psicóticos e agressividade durante os atendimentos. Fazuso de Risperidona. ${ }^{\circledR}$

Genitora relata que o paciente tinha uma vida normal até os 21 anos, quando começou a cair para trás e apresentar pé equino. Foi, então, encaminhado ao neurologista, que solicitou ressonância magnética, comprovando o sinal do olho de tigre, característico da doença. Em seguida, foi internado por um mês no hospital Sarah Kubitschek para acompanhamento, tendo sido encaminhado para tratamento fisioterápico posteriormente.

Durante a avaliação, observou-se dificuldade de deambular, presença de órteses em ambas as pernas, movimentos involuntários e disartria. À palpação, apresentou rigidez muscular em membros superiores (MMSS) e inferiores (MMII), encurtamento de isquiotibiais e contraturas em flexores plantares (gastrocnêmios e sóleo) e dorso-flexores (tibial anterior), com grau de força normal nos demais músculos.

A mobilização mostrou posteriorização corporal à deambulação, pé equino (uso de órteses), movimentos coreoatetósicos, dificuldade de pegar objetos. Apresentou ainda clônus e sinal de Babinski presente, sinal de cano de chumbo, alteração no equilíbrio dinâmico, hipertonia espástica e hiperreflexia patelar.

A perimetria ${ }^{8}$ de membro superior teve como base a interlinha do cotovelo, com duas marcações, de 10 e 20 centímetros (cm) acima e abaixo (Tabela 1). Já no membro inferior a base foi a região da fossa poplítea, com três marcações, de 10, 20 e 30 $\mathrm{cm}$, acima e abaixo (Tabela 2).

A goniometria9 foi realizada na articulação do tornozelo, a mais afetada. As demais articulações apresentavam amplitudes de movimento normais (Tabela 3).

O protocolo de exercícios físicos teve como objetivo reeducação da marcha, fortalecimento muscular de MMSS e MMII, melhora no equilíbrio e expansão pulmonar, no intuito de proporcionar melhora do quadro clínico do paciente.

Tabela 1. Perimetria de membros superiores.

\begin{tabular}{lll}
\hline $\begin{array}{l}\text { Marcações da } \\
\text { de MMSS }\end{array}$ & $\begin{array}{l}\text { perimetria } \\
\text { direito }\end{array}$ & $\begin{array}{l}\text { Membro superior } \\
\text { esquerdo }\end{array}$ \\
\hline $20 \mathrm{~cm}$ acima & $27 \mathrm{~cm}$ & $26 \mathrm{~cm}$ \\
$10 \mathrm{~cm}$ acima & $25 \mathrm{~cm}$ & $24 \mathrm{~cm}$ \\
$10 \mathrm{~cm}$ abaixo & $23,5 \mathrm{~cm}$ & $22,5 \mathrm{~cm}$ \\
$20 \mathrm{~cm}$ abaixo & $17 \mathrm{~cm}$ & $15 \mathrm{~cm}$ \\
\hline
\end{tabular}

MMSS: membros superiores. 
Tabela 2. Perimetria de membros inferiores.

\begin{tabular}{lll}
\hline $\begin{array}{l}\text { Marcações da } \\
\text { perimetria de MMII }\end{array}$ & $\begin{array}{l}\text { Membro inferior } \\
\text { direito }\end{array}$ & $\begin{array}{l}\text { Membro inferior } \\
\text { esquerdo }\end{array}$ \\
\hline $30 \mathrm{~cm}$ acima & $47,5 \mathrm{~cm}$ & $46 \mathrm{~cm}$ \\
$20 \mathrm{~cm}$ acima & $41,5 \mathrm{~cm}$ & $40 \mathrm{~cm}$ \\
$10 \mathrm{~cm}$ acima & $34,5 \mathrm{~cm}$ & $33,5 \mathrm{~cm}$ \\
$10 \mathrm{~cm}$ abaixo & $29,5 \mathrm{~cm}$ & $31 \mathrm{~cm}$ \\
$20 \mathrm{~cm}$ abaixo & $27 \mathrm{~cm}$ & $25 \mathrm{~cm}$ \\
$30 \mathrm{~cm}$ abaixo & $21 \mathrm{~cm}$ & $20 \mathrm{~cm}$ \\
\hline
\end{tabular}

MMII: membros inferiores.

Tabela 3. Goniometria de tornozelo.

\begin{tabular}{lll}
\hline & Pé direito & Pé esquerdo \\
\hline Dorso-flexão & $05^{\circ}$ & $00^{\circ}$ \\
Flexão plantar & $15^{\circ}$ & $20^{\circ}$ \\
\hline
\end{tabular}

Os atendimentos começaram com alongamento de MMII e mobilização articular de tornozelo e joelho, seguindo-se com crioestimulação na planta do pé para ganho de dorso-flexão, no começo do movimento o paciente fazia ativamente e sendo o movimento complementado passivamente.

Utilizou-se Estimulação Elétrica Funcional (FES) ${ }^{10}$ em flexores plantares e dorso-flexores para ganho de força muscular, inicialmente, revertendo-se essa aplicação apenas para dorso-flexores devido a melhor resposta do paciente.

A partir da terceira semana, acrescentou-se treino muscular em plataforma vibratória, estando o paciente em bipedestação, associando-se exercícios de cadeia cinética fechada, com 5 séries de 12 repetições para flexão plantar e dorso-flexão. No intervalo das séries a plataforma não era desligada, no total o paciente permanecia 5 minutos. A Facilitação Neuromuscular Proprioceptiva (FNP) visou à melhora da resposta neuromuscular, sendo realizada em MMII, nos padrões extensor e flexor, nas diagonais funcional e primitiva.

O treinamento de equilíbrio deu-se no disco de propriocepção. Já o treino de marcha ocorreu nas barras paralelas, com a marcha de Tundem, estando o paciente com olhos abertos e depois fechados, para maior integração sensorial.

Os resultados obtidos foram satisfatórios, com melhora na deambulação e no equilíbrio, ganho de amplitude de movimento em flexão plantar de ambos os tornozelos, alívio na contratura de músculos posteriores da coxa, diminuição dos espasmos, controle mais amplo nos movimentos e capacitação para as atividades diárias.

A crioestimulação pode promover redução da atividade elétrica muscular e consequente melhora no desempenho funcional, ${ }^{11}$ aperfeiçoando, portanto, a percepção e a realização do movimento, como observado no ganho de dorso-flexão do referido paciente.

A literatura relata que, após realização de exercícios de alta intensidade na plataforma vibratória, constata-se melhora na função motora, na capacidade física, no equilíbrio e na percepção de fadiga, ${ }^{12}$ corroborando, assim, a presente pesquisa, na qual o paciente obteve fortalecimento motor, mediante elevação da amplitude de força com tal treinamento.

A estimulação elétrica funcional (FES) utiliza corrente elétrica de baixa frequência para provocar contração de músculos paralisados ou enfraquecidos decorrentes de lesão do neurônio motor superior. ${ }^{13}$ Tal aparato foi aqui empregado por possibilitar a contração muscular funcional, por estimulação elétrica, despolarizando o nervo motor e produzindo resposta sincrônica em todas as unidades do músculo, permitindo movimentos úteis à locomoção.

A FNP promove reaquisição de habilidades de movimentos perdidos ou com hipofunção, levando em consideração o processo de neuroplasticidade, proporcionando maior autonomia e independência em pacientes com traumas neurológicos, ${ }^{14}$ técnica utilizada nesse relato como terapia de suporte, com o fito de aprimorar informações somatossensoriais para a melhora do equilíbrio corporal.

O treinamento do equilíbrio pode induzir neuroplasticidade estrutural, por intermédio do desafio aos sistemas de percepção vestibular e sensório-motor, comprovada pelo aumento da espessura cortical em regiões específicas de associação visual e equilíbrio. ${ }^{15}$ Nesse sentido, propõe-se que o exercício com disco de propriocepção, realizado nesse estudo, induza uma melhora do desempenho do equilíbrio e da deambulação, como observado no paciente em questão, promovendo, portanto, uma maior estabilidade do centro de gravidade deste.

\section{CONCLUSÃO}

Apesar de rara, estudos científicos sobre a síndrome de Hallervorden-Spatz direcionam para o importante papel dos exercícios físicos no ganho motor desses pacientes. O relato de caso em questão usou como terapêutica exercícios físicos para melhora de deambulação, coordenação, equilíbrio e atividades diárias, com resultados satisfatórios. Evidencia-se, ainda, a relevância de mais estudos na literatura que elucidem protocolos de exercícios como forma de treinamento físico e melhora do quadro clínico do paciente com síndrome de Hallervorden-Spatz. 


\section{REFERÊNCIAS}

1. Hallervorden J, Spatz H. Eigenartige erkrankung im extrapyramidalen system mit besonderer beteiligung des globus pallidus und der substantia nigra: ein beitrag zu den beziehungen zwischen diesen beiden zentren. Z Ges Neurol Psychiat. 1922;79:254302.

2. Mehta P, Singh A, Gathwala G, Rohilla S. Hallervorden spatz syndrome: eye of the tiger. J Pediat Sci. 2016;8:e250.

3. McNeill A, Birchall D, Hayflick SJ, Gregory A, Schenk JF, Zimmerman EA, et al. T2* and FSE MRI distinguishes four subtypes of neurodegeneration with brain iron accumulation. Neurology. 2008;70(18):1614-9.

4. Hayflick SJ, Westaway SK, Levinson B, Zhou B, Johnson MA, Ching KH, et al. Genetic, clinical, and radiographic delineation of Hallervorden-Spatz syndrome. N Engl J Med. 2003; 348(1):3340 .

5. Cavalcante MO, Carvalho AL Júnior, Alencar FI Neto, Almeida DM. A síndrome de Hallervorden Spatz e sua patogênese: considerações sobre o determinismo genético. Rev Interd Ciên Saúde. 2017;4(2):53-60.

6. Gregory A, Hayflick SJ. Genetics of neurodegeneration with brain iron accumulation. Curr Neurol Neurosci Rep. 2011;11(3):254-61.

7. Díez ME, Puy JF. Fisioterapia del Síndrome de HallervordenSpatz. A propósito de un caso [Internet]. Zaguan: Universidad de Zaragoza Repository; 2012[acesso em: 25 mar 2020]. Disponível em: https://zaguan.unizar.es/record/7342/files/TAZ-TFG-2012-171. pdf

8. Ferraresi C, Brito OT, Oliveira ZL, Menezes RR, Baldissera V, Andrade PS, et al. Effects of low level laser therapy $(808 \mathrm{~nm})$ on physical strength training in humans. Lasers Med Sci. 2011;26(3):34958 .

9. Fraser JJ, Koldenhoven RM, Saliba SA, Hertel J. Reliability of ankle-foot morphology, mobility, strength, and motor performance measures. Int J Sports Phys Ther. 2017;12(7):1134-49.

10 Jones S, Man WD, Gao W, Higginson IJ, Wilcock A, Maddocks M. Neuromuscular electrical stimulation for muscle weakness in adults with advanced disease. Cochrane Database Syst Rev. 2016;10(10):CD009419.

11. Felice TD, Ishizuka RO, Amarilha JD. Eletroestimulação e crioterapia para espasticidade em pacientes acometidos por acidente vascular cerebral. Revista Neurociências. 2011;19(1):77-84.

12. Bergmann G, Kutzner I, Bender A, Dymke J, Trepczynski A, Duda GN, et al. Loading of the hip and knee joints during whole body vibration training. PLoS One. 2018;13(12):e0207014.

13. Bergmann M, Zahharova A, Reinvee M, Asser T, Gapeyeva $\mathrm{H}$, Vahtrik D. The effect of functional electrical stimulation and therapeutic exercises on trunk muscle tone and dynamic sitting balance in persons with chronic spinal cord injury: a crossover trial. Medicina (Kaunas). 2019;55(10):619.

14. Nakada CS, Meningroni PC, Ferreira AC, Hata L, Fuzaro AC, Marques W Júnior, et al. Ipsilateral proprioceptive neuromuscular facilitation patterns improve overflow and reduce foot drop in patients with demyelinating polyneuropathy. J Exerc Rehabil. 2018;14(3):503-8.

15. Rogge AK, Röder B, Zech A, Hötting K. Exercise-induced neuroplasticity: Balance training increases cortical thickness in visual and vestibular cortical regions. Neuroimage. 2018;179:471-9.

\section{Como citar:}

Almeida LM, Belchior LD. Treinamento físico de paciente com a síndrome de Hallervorden-Spatz: um relato de caso. Rev Med UFC. $2021 ; 61(1): 1-4$. 